\title{
ORGANIZACIÓN DE LOS SERVICIOS DE PROTECCIÓN A LA INFANCIA EN LA COMUNIDAD VALENCIANA. ANÁLISIS NORMATIVO Y FUNCIONAL
}

\section{JOSÉ CONRADO MOYA MIRA}

Psicólogo. Jefe de la Sección de Familia, Infancia y Juventud de la Dirección Territorial de Bienestar Social de Alicante.

\section{INTRODUCCIÓN}

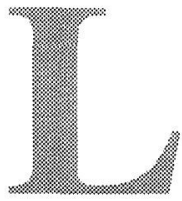

a Constitución Española de 1978 supone el punto de arranque de una nueva concepción de las responsabilidades que, respecto de la protección a la infancia, asumen las Entidades Públicas en nuestro país.

Esta nueva orientación atribuye un papel no sustitutivo de la función paterna y materna, sino que atribuye la responsabilidad de asegurar la protección a la infancia cuando se produzcan situaciones en las que los niños queden o estén en riesgo de quedar en situación de desamparo o desprotección, debiendo disponer los mecanismos necesarios para corregir tal situación.

Así, en el artículo 39 de la Constitución Española, se dice textualmente:

«1. Los poderes públicos asegurarán la protección social, económica y jurídica de la familia.

2. Los poderes públicos asegurarán también la protección integral de los hijos, iguales ante la ley, con independencia de la filiación y de las madres, sea cual sea su estado civil. La Ley hará posible la investigación de la paternidad.

3. Los padres han de prestar asistencia completa a los hijos tenidos dentro o fuera del matrimonio, durante la minoría de edad y en los otros casos en que la Ley obligue.

4. Los niños gozarán de la protección prevista en los acuerdos internacionales que velen por sus derechos».

Se deja en manos de la Entidad Pública competente la adopción de medidas para garantizar la protección de la infancia.

Esta capacitación de las Entidades Publicas para la adopción de medidas de protección se complementa con lo preceptuado en la 
Constitución cuando se posibilita que las distintas Comunidades Autónomas puedan asumir competencias plenas en materia de protección de menores.

En el caso de la Comunidad Autónoma Valenciana, la entrada en vigor del Estatuto de Autonomía (1982) da paso a un proceso de transferencias en materia de servicios sociales y, por tanto, de protección a la infancia, asumiendo en la actualidad competencias plenas en esta materia.

\section{ESTRUCTURA ORGÁNICA Y PROTECCIÓN A LA INFAN- CIA}

El proceso progresivo de asunción de competencias, por parte de las Entidades Públicas de Comunidades Autónomas, iniciado, en el caso de la Comunidad Valencia, en 1982, supone la recepción no sólo de centros y servicios, sino de los organismos por los que venían siendo gestionados, por ejemplo, las Juntas de Protección de Menores, el I.N.A.S., etc.

La necesidad de integrar en una estructura orgánica única todas las competencias en materia de Servicios Sociales y de Protección a la Infancia ha constituido un criterio asumido por la Generalitat Valenciana que, utilizando distintos modelos organizativos (Dirección General, Instituto Valenciano de Servicios Sociales, etc.), atribuye en la competencia exclusiva en materia de protección y guarda de menores a la Consellería de Bienestar Social.

De forma simultánea a la creación de una estructura única, se inicia un proceso de descentralización y desconcentración, que supone la organización de competencias relacionadas con los Servicios Sociales y con la Protección a la Infancia, atendiendo a dos niveles:

1. El nivel de Atención Primaria, que supone intervenciones de carácter preventivo y generalizado y cuya competencia es asumida por los Ayuntamientos o agrupaciones de Ayuntamientos.

2. El nivel de Atención Especializada, que supone intervenciones técnicas y complejas cuya competencia es asumida por la Generalitat y por algunos Ayuntamientos, cuando el ámbito de actuación sea local.

Este principio de descentralización se combina con el de globalización de la intervención, fijándose tres modalidades de intervención que pretenden configurar un modelo continuo basado en el desarrollo de medidas de carácter preventivo, asistencial y rehabilitador. 


\section{III.EL PAPEL DE LAS ENTIDADES PÚBLICAS CONFORME LA NORMATIVA BÁSICA DE PROTECCIÓN A LA INFANCIA A NIVEL ESTATAL.}

\subsection{LA LEY 21/87, de 11 de noviembre, por la que se modifican de- terminados artículos del Código Civil y de la Ley de Enjuicia- miento Civil en materia de adopción.}

La Ley 21/87, de 11 de noviembre, por la que se modifican determinados artículos del Código Civil y de la Ley de Enjuiciamiento Civil en materia de adopción, va a suponer una profunda transformación no sólo en la regulación de la adopción sino en el establecimiento de nuevas instituciones que inciden profundamente en la protección a la infancia, así como en lo referente a la organización competencial en esta materia.

La actuación para garantizar la protección a los menores en situación de desamparo (definido en el art. 172 del C.C.) pasa a ser competencia de las Entidades Públicas, a las que, en el respectivo territorio esté encomendada la protección de Menores, pudiendo ésta asumir la tutela de los mismos por ministerio de la ley, pasando el control judicial a un segundo término en caso de que fuera necesario la resolución de conflictos producidos por esta primera actuación.

Con la entrada en vigor de la Ley $21 / 87$, se produce una modificación muy importante de orden competencial, dado que queda suprimida la llamada «facultad protectora» de los Tribunales Tutelares de Menores, quedando ésta asumida por la Administración Autonómica.

Esta asunción de competencias por parte de la Entidad Pública Autonómica no supone la derogación expresa de la Ley de Tribunales Tutelares de Menores de 1948, cuestión que ha quedado resuelta con la entrada en vigor de la Ley Orgánica 1/96 de Protección Jurídica del Menor que, en su Disposición derogatoria única, dice textualmente: "Queda derogado el Decreto de 2 de julio de 1948 por el que se aprueba el texto refundido de la legislación sobre Protección de Menores y cuantas normas se opongan a la presente Ley".

Igualmente, atribuye al Ministerio Fiscal la superior vigilancia de la tutela, guarda o acogimiento resueltos por la Entidad Pública.

En cuanto a los principios que deben informar la actuación de las Entidades Públicas competentes en protección de menores, se establecen:

- El carácter prioritario del interés del menor.

- La necesidad de mantener al menor en su medio familiar siempre que sea posible. 
- La configuración de las instituciones de guarda, acogimiento y adopción como instrumentos de integración familiar.

- La utilización del recurso de internamiento sólo cuando sea indispensable y por el menor tiempo posible.

3.2. LA LEY ORGANICA 1/96, de 15 de enero (B.O.E. 17 de enero de 1996) de Protección Jurídica del Menor, de modificación parcial del Código Civil y de la Ley de Enjuiciamiento Civil.

La Ley Orgánica 1/96 viene a intentar dar respuesta a las lagunas que la aplicación de la Ley 21/87 había puesto de manifiesto en sus ocho años de aplicación. Se aborda una reforma profunda de las instituciones de protección a la infancia partiendo del reconocimiento del menor de edad como sujeto participativo y activo, incidiendo en la idea de que la mejor forma de garantizar la protección a la infancia es promover su autonomía como sujetos.

La Ley Orgánica 1/96 establece los principios rectores que deben regular la actuación de la Entidad Pública, definiendo los siguientes:

1. La supremacía del interés del menor.

2. El mantenimiento del menor en el medio familiar de origen, salvo que no sea conveniente para su interés.

3. Su integración social y familiar.

4. La prevención de todas aquellas situaciones que puedan perjudicar su desarrollo personal.

5. Sensibilizar a la población ante situaciones de indefensión del menor.

6. Promover la participación y solidaridad social.

7. La objetividad, imparcialidad y seguridad jurídica en la actuación protectora, garantizando el carácter colegiado e interdisciplinar en la adopción de las medidas.

El artículo 14 de la L.O. 1/96 determina, igualmente, el principio de colaboración, que obliga a que "en toda intervención, se procurará contar con la colaboración del menor y de su familia y no interferir en su vida escolar, social o laboral".

En relación a las actuaciones de la Entidad Pública ante situaciones de desprotección social del menor, se definen dos tipos de situaciones:

a. Situación de riesgo. Definida como aquélla que pueda perjudicar el desarrollo personal o social del menor que NO requieran de la asunción de tutela por parte de la Entidad Pública.

b. Situación de desamparo. Definida como aquélla que se produce de hecho, a causa del incumplimiento, o del imposible o inadecuado ejercicio, de los deberes de protección establecidos por las leyes 
para la guarda de los menores, cuando éstos queden privados de la necesaria asistencia moral o material.

Ante las situaciones de riesgo, la Entidad Pública debe poner en marcha las actuaciones pertinentes para reducirlas y realizar el seguimiento de la evolución del menor en la familia.

Ante las situaciones de desamparo, la Entidad Pública debe adoptar las medidas de protección, asumiendo la tutela del menor/es y poniéndolo en conocimiento del Ministerio Fiscal.

La Entidad Pública asumirá la guarda del menor bien cuando previamente haya sido declarado en desamparo o bien a solicitud de los padres, cuando concurran circunstancias graves que impidan a aquéllos el cuidado de su/s hijos/s o en los casos en que sea determinado por el Juez competente.

La guarda de un menor podrá ser ejercida a través de las siguientes figuras:

A. ACOGIMIENTO FAMILIAR, que podrá adoptar las siguientes modalidades:

1. Acogimiento familiar simple: de carácter transitorio, bien porque se prevea la reinserción al núcleo familiar de origen bien hasta garantizar una medida de protección más estable.

2. Acogimiento familiar permanente, cuando la edad u otras circunstancias del menor así lo aconsejen.

3. Acogimiento familiar preadoptivo, formalizado por la Entidad Pública cuando eleve propuesta de adopción, o considere necesario establecer un periodo de adaptación del menor a la familia.

4. Acogimiento familiar provisional, posibilitando que la Entidad Pública resuelva en interés del menor, en los casos de no consentimiento u oposición de los padres, el acogimiento familiar en tanto se produce una resolución judicial.

B. ACOGIMIENTO RESIDENCIAL, procediendo al internamiento de un menor, procurando que tenga la menor duración posible, salvo que convenga al interés del menor.

Los centros u Hogares funcionales deberán estar autorizados y acreditados por la Entidad Pública, debiendo prestar especial atención a la seguridad, sanidad, número y cualificación profesional de su personal, proyecto educativo, participación de los menores en su funcionamiento interno y demás condiciones que contribuyan a asegurar sus derechos.

Se prestará especial atención a la obligación establecida en el artículo 22 de la Ley Orgánica 1/96 de informar a los padres, tutores o guardadores sobre la situación de los menores internos, cuando no exista Resolución Judicial que lo prohiba. 
C. $L A A D O P C I O N$. La tramitación de la adopción tanto nacional como internacional compete a las Entidades Públicas, pudiendo delegar determinadas funciones de mediación a Entidades acreditas.

En el caso de la Adopción Internacional, se estará a lo dispuesto en el Convenio Relativo a la Protección del Niño y a la cooperación en materia de adopción internacional (Convenio de la Haya).

No obstante esto, y a expensas de la regulación por parte de las Comunidades Autónomas de normativa que regule la acreditación de las Entidades de Mediación en materia de adopción Internacional, la Ley Orgánica 1/96 determina las funciones que podrán ser asumidas por éstas y que serán:

1. Información y asesoramiento a los interesados en materia de adopción internacional.

2. Tramitación de expedientes ante las autoridades españolas y extranjeras.

3. Asesoramiento y apoyo a los solicitantes de adopción.

Las Entidades Públicas deberán asegurar, en todo caso:

1. La recepción y tramitación de solicitudes, ya sea directamente o a través de Entidades acreditadas.

2. La expedición de los certificados de idoneidad y compromiso de seguimiento en los países que se requiera.

3. La acreditación, control, inspección y elaboración de directrices de actuación de las Entidades que realicen funciones de mediación en sus respectivos territorios.

La emisión de los certificados de idoneidad se realizarán a partir de la elaboración de los preceptivos informes psicosociales, pudiendo incorporar procesos de formación de familias que así lo deseen.

Indicar a este respecto que en la tramitación de Adopción Internacional hay países que ponen como requisito la existencia de un Certificado de acreditación de haber recibido formación, por lo que la Dirección General de Servicios Sociales ha implantado los referidos cursos a partir de septiembre de 1996.

IV.NORMATIVA BÁSICA DE PROTECCION DE MENORES A NIVEL DE LA COMUNIDAD AUTONOMA VALENCIANA.

Con anterioridad a la publicación y entrada en vigor de la Ley $21 /$ 87 en la Comunidad Valenciana, se había regulado una modalidad de acogimiento familiar con finalidad de retorno, conocido como familias educadoras. 
Igualmente, se publica el llamado Libro Blanco del Menor (1987) que pretende organizar las prestaciones, servicios y recursos dispuestos para la protección a la infancia de una manera eficaz.

En la Comunidad Valenciana, la Ley 21/87 fue desarrollada a través del Decreto 23/88 sobre medidas de protección de menores desamparados, modificado parcialmente con posterioridad por el Decreto $31 / 91$.

El Decreto 23/88 establece los criterios que orientan la actuación de la Administración Pública Valenciana en materia de protección de menores en situación de desamparo, los procedimientos que regirán la actuación de la Administración a fin de evitar la arbitrariedad de los Poderes Públicos y regula las medidas aplicables para garantizar las protección de menores.

Como consecuencia del desarrollo legislativo llevado a cabo tanto a nivel estatal como autonómico, el Gobierno Valenciano considera necesario dar rango de Ley a toda la normativa que de una manera dispersa había ido regulando el sector de infancia.

De esta manera se publica en D.O.G.V. de 16-12-94 la Ley 7/94 de la Infancia de la Generalitat Valenciana, que tiene por objeto ordenar las competencias atribuidas a la Generalitat Valenciana, tanto en materia de protección de menores como en reforma de menores.

Así, se desarrollan los programas de atención a la infancia que deberán ser implantados para el cumplimiento de estas competencias, siguiendo los principios y criterios organizativos que a continuación se exponen.

\section{PROGRAMAS DE PROTECCIÓN A LA INFANCIA EN LA COMUNIDAD VALENCIANA.}

Como ya he indicado anteriormente, los programas de protección a la infancia vigentes en la Comunidad Autónoma Valenciana se rigen por una organización que distingue dos niveles de intervención:

1. El Nivel de Atención Primaria, recayendo la responsabilidad de su implantación en los Ayuntamientos, a través de los Servicios Sociales Generales.

2. El Nivel de Atención Especializada, competencia de la Generalitat Valenciana y de los Ayuntamientos, en los casos recogidos en la Ley de Servicios Sociales.

Estos niveles de intervención estructuran secuencias de prestaciones y servicios que configuran programas en torno a una concepción de necesidades de la infancia agrupadas en cinco grandes esferas: 
1. Necesidades de información.

2. Necesidades de accesibilidad.

3. Necesidades de cooperación.

4. Necesidades de convivencia.

5. Necesidades de reinserción.

Sin entrar detalladamente a cada uno de ellos enunciaré el desarrollo previsto en la Ley $7 / 94$.

\subsection{Programas de información.}

a. Nivel Primario:

- Campañas informativas y de sensibilización.

- Servicios de asesoramiento y primer diagnóstico.

b. Nivel Especializado:

- Campañas autonómicas de información y sensibilización.

- Servicios de diagnóstico especializado.

\subsection{Programas de accesibilidad.}

a. Nivel Primario:

- Seguimiento del absentismo escolar.

- Promoción de la salud infantil.

- Fomento de la inserción prelaboral.

b. Nivel Especializado:

- Prevención de los malos tratos.

- Integración escolar.

- Medidas laborales de inserción.

- Eliminación de barreras arquitectónicas y de comunicación.

\subsection{Programas de cooperación.}

a. Nivel Primario:

- Promoción de la autoayuda.

- Fomento del voluntariado social.

- Promoción de organizaciones solidarias.

b. Nivel Especializado:

- Acreditación de centros y servicios.

- Regulación del régimen de cooperación.

- Reconocimientos de las instituciones colaboradoras de integración familiar. 


\subsection{Programas de convivencia.}

a. Nivel Primario:

- Ayuda familiar (económica, educativa, etc.).

- Acogimiento familiar de carácter primario (f. educadora/ext.).

b. Nivel Especializado:

- La terapia familiar.

- El acogimiento familiar de carácter especializado.

- El acogimiento en Residencias.

- La emisión de Propuestas previas para adopción.

\subsection{Programas de reinserción.}

Estos programas van dirigidos a jóvenes que presentan problemas de inadaptación y que se encuentran cumpliendo medida judicial dictada por el Juzgado de Menores.

En su desarrollo entraremos con posterioridad, cuando tratemos la intervención de la Entidad Pública en materia de ejecución de medidas judiciales impuesta a jóvenes infractores.

De las secuencias de prestaciones y servicios que permiten el desarrollo de Programas de Atención a la Infancia, se puede concluir que la Ley no entiende la protección a la infancia como la implantación de un sistema dicotomizado en medidas de internamiento o de acogimiento familiar, sino que, por el contrario, se estructura un continuo de programas que garanticen una estructura sólida de Prevención, asistencia y reinserción.

En el desarrollo de estos Programas no sólo está implicado y reponsabilizado el sistema de Servicios Sociales, sino que se reconoce como indispensable la integración y articulación de las acciones promovidas desde el resto de sistemas de atención a la infancia (Educación, Sanidad, ocio y tiempo libre, etc.).

Así, en el Preámbulo de la Ley 7/94, se dice textualmente:

«Las nuevas necesidades de los niños, la calidad de los servicios de la infancia y la promoción de nuevas oportunidades requieren de una articulación adecuada de las administraciones educativas, sanitarias, sociales, culturales y de preparación al mundo laboral.» 


\section{VI.PRINCIPIOS QUE ORIENTAN LA ORGANIZACIÓN DE RE- CURSOS PARA EL DESARROLLO DE PROGRAMAS DE PRO- TECCIÓN A LA INFANCIA.}

Para entender la organización e implantación actual de los recursos dispuestos para promover el desarrollo de los Programas de Protección a la Infancia es necesario analizar su proceso de configuración íntimamente relacionado con el desarrollo de los Servicios Sociales en nuestra Comunidad Autónoma.

Así, los recursos con los que la Generalitat Valenciana contaba en el momento de asumir las competencias de protección de menores se reducían a dos posibilidades:

1. El mantenimiento del niño/a en su domicilio con algunos apoyos de índole económica (en el mejor de los casos).

2. El ingreso en un centro de internamiento.

De forma paulatina se operaron cambios profundos en el sistema de protección a la infancia que pasaban necesariamente por:

1. Objetivar los procedimientos de valoración de solicitudes de utilización de servicios especializados.

2. Ampliar los recursos disponibles en la red básica de Servicios Sociales (Atención Primaria), de forma que fuera posible desarrollar y diversificar actuaciones preventivas eficaces y sistemas de detección precoz de los casos.

3. La mejora de las prestaciones de los centros de infancia a partir de cambios que afectarán a la organización, infraestructura, profesionalización...

4. El desarrollo de los programas de acogimiento familiar dispuestos, tanto con finalidad de retorno al núcleo familiar de origen como de proporcionar una alternativa familiar a menores pertenecientes a familias cuyo pronóstico de recuperabilidad fuese negativo.

5. La diversificación de otros programas de atención especializada que fueran capaces de no bipolarizar el tipo de respuesta (acogimiento familiar-acogimiento residencial) ofertada a la infancia en situación de desprotección social.

Entraré en el desarrollo de los puntos concernientes al acogimiento residencial.

\subsection{Objetivar procedimientos de utilización de servicios especiali- zados.}

Las medidas de protección a la infancia hasta principios de la década de los años 80 estaban basadas fundamentalmente en medidas de 
internamiento o de mantenimiento en domicilio con algunos apoyos económicos.

Los niños/as eran acogidos en instituciones de forma poco objetiva, produciéndose una falta de rigurosidad en los procesos de valoración de problemáticas que aconsejaban el internamiento. De esta forma, podíamos encontrarnos en los centros menores cuyo único problema familiar era la falta de recursos económicos, junto a otros cuyas problemáticas eran mucho más agudas (malos tratos, abandono, etc.).

La implantación progresiva de la red de atención primaria de nivel municipal, el establecimiento de equipos de recepción de nivel provincial capaces, los primeros, de detectar, prevenir y prestar un primer nivel de asistencia generalizado a las situaciones de riesgo en las que pudiera encontrarse un menor $\mathrm{y}$, los segundos, de objetivar y unificar criterios de utilización de recursos de nivel especializado, han provocado efectos positivos, entre los que destacaría:

- Una mayor especialización de los equipos de atención primaria que trabajan con infancia (todavía no suficiente).

- Reducción del número de plazas de internado.

- Fomento de programas de apoyo a la familia, con carácter preventivo.

Igualmente, y como en cualquier proceso, se detectan efectos no deseables, consecuencia de la aplicación de los nuevos criterios de tratamiento de la problemática infantil. Entre ellos, destacaría:

- La mayor complejidad de los casos que llegan a los internados, dado que se atienden casos que han experimentado situaciones de grave deprivación, abandono o maltrato en sus propias familias.

- El agrupamiento de menores que acceden a los internamientos en dos bloques de edades, primera infancia y adolescentes.

- Aparición de nuevas problemáticas, como, por ejemplo, el consumo de tóxicos, agresividad y falta de control extremo, absentismo escolar persistente, aislamiento y dificultad para responder a los demás, etc.

De forma paulatina, los acogimientos residenciales pierden su carácter de contraposición al resto de posibles medidas a adoptar y entra a formar parte de un continuo de alternativas posibles que, además, es recomendable para determinado tipo de problemáticas que no pueden mantenerse en acogimientos familiares o en familia biológica.

En definitiva, la mejora de los procesos de objetivación y valoración de casos de menores que puedan encontrarse en situación de riesgo o desamparo es un objetivo permanente, necesario, para: 
1. Dar cumplimiento a lo dispuesto en el art. 11 y 16 de la Ley Orgánica 1/96 que establece "que la Entidad Pública regirá su actuación garantizando la objetividad, imparcialidad y seguridad jurídica en la actuación protectora, garantizando igualmente el carácter colegiado e interdisciplinar en la adopción de las medidas.

2. Que la Entidad Pública deberá verificar la situación denunciada y adoptará las medidas necesarias para resolverla en función del resultado de aquella.

3. Posibilitar la utilización de los recursos y prestaciones que a cada caso convenga, individualizando en lo posible, el tratamiento del caso".

\subsection{Ampliación de recursos en el nivel de atención primaria.}

El desarrollo del sistema de protección a la infancia ha estado íntimamente relacionado con la implantación de la red de Servicios Sociales en nuestro país.

El criterio de municipalización de los recursos ha sido determinante en la organización y desarrollo de los Servicios Sociales y, por tanto, del sistema de protección infantil.

Con su aplicación, se ha dado una importancia relevante al desarrollo del nivel de atención primaria de los servicios sociales. Este nivel de atención primaria garantizará la implantación de las llamadas prestaciones básicas de carácter polivalente y generalizado.

El carácter polivalente de la atención primaria pretende acabar con la extrema sectorización de la red de servicios sociales existentes en los primeros años de la década de los ochenta.

La implantación de los Servicios Sociales de nivel primario es potenciado desde las propias Comunidades Autónomas con la colaboración de las Entidades locales y reciben un impulso definitivo con la implantación, en el año 1988 y por parte del Ministerio de Asuntos Sociales, del llamado Plan Concertado.

Las medidas previstas dentro de los distintos programas a nivel de atención primaria pretenden un triple objetivo:

a. Garantizar el acceso al sistema de servicios sociales de la población en general, así como la detección temprana de casos que se encuentren en posible situación de desprotección.

b. Garantizar la implantación de un nivel de prestaciones básicas que aseguren la atención de los usuarios sin necesidad de separación innecesaria del núcleo familiar de convivencia.

c. Elaborar los informes técnicos pertinentes que diagnostican y fundamentan una solicitud de atención del caso desde los servicios de nivel especializado. 
En el nivel de atención primaria, aparece, como especialmente necesario, la implicación de los sistemas que intervienen en la protección infantil, sobre todo de Educación, Sanidad, Servicios Sociales, así como de otros organismos judiciales, o incluso del ámbito policial, que asegure la rapidez en las actuaciones y la objetividad e imparcialidad que debe regir en la actuación de las Entidades Públicas en materia de protección de menores.

Respecto de los tres objetivos que se pretenden con el desarrollo del nivel de atención primario de protección infantil, podríamos indicar lo siguiente:

- Que comienzan a estructurarse fórmulas de coordinación intersistemas que permiten la detección precoz de situaciones de riesgo o desamparo, aunque sigue siendo necesario ampliar el conocimiento que respecto de los procedimientos de protección a la infancia tienen los ciudadanos en general y los profesionales en particular.

- La existencia de equipos técnicos interdisciplinares garantizan procedimientos ágiles de elaboración de diagnósticos que asegurando la objetividad de las medidas propuestas a adoptar con los menores, resulten ágiles y eficaces.

- Sin embargo, el nivel de asistencia a casos, promoviendo medidas que den respuesta integral a la situación de desprotección en la que se encuentra o puede encontrarse un niño, procurando su integración familiar, es, en mi opinión, todavía muy insuficiente y debe ser el objetivo básico a conseguir en los próximos años.

El insuficiente desarrollo de los servicios de intervención familiar, desde el nivel primario de actuación, provoca, en ocasiones, el acceso innecesario de algunos casos a servicios de nivel especializado.

Las medidas de atención primaria han sido dispuestas de forma jerárquica, así, en lo referente a los Programas de Convivencias, se han dispuesto medidas que van desde los apoyos económicos a la familia hasta programas de acogimiento temporal con finalidad de retorno al núcleo familiar de origen (acogimiento provisional según L.O. 1/96), pasando por los programas de intervención familiar de carácter educativo o de ayuda a domicilio.

Sin embargo, denotamos que estos programas han recaído sobre los Equipos Municipales de Servicios Sociales Generales que tienen como encargo la implantación del resto de medidas previstas con carácter general y polivalente para todos los sectores de población.

Este hecho provoca que no exista la necesaria especificidad en el desarrollo de los programas de nivel primario destinados a la protección a la infancia y se produzcan situaciones no deseadas como las anteriormente referidas. 
Es por ello que parece oportuno potenciar la creación de un nivel intermedio entre la atención primaria y la atención especializada capaz de abordar situaciones específicas de menores en situación de riesgo, diversificando de esta manera la secuencialización de prestaciones y servicios.

\subsection{La mejora en la organización y prestaciones de los centros.}

Respecto del trabajo realizado en centros de infancia, ha pasado por abordar procesos de normalización de la atención a los menores allí atendidos, evitando la dinámica de Institución Total y procurando la incorporación a los servicios de la redes ordinarias de Educación, Sanidad, Servicios Sociales, culturales, etc.

Se han realizado actuaciones dirigidas a mejorar la infraestructura de los centros, las dotaciones de personal, la diversificación de tipologías de centros y, en definitiva, la delimitación de condiciones necesarias que debe cumplir un Centro para autorizar su funcionamiento en el territorio de la Comunidad Valenciana. Para ello, se desarrolló normativamente el llamado plan de ordenación de los servicios sociales de la Comunidad Valenciana, en los que quedaban incorporados los centros y servicios de atención a la infancia.

Se aborda el desarrollo de Proyectos Educativos, Regalamentos Régimen Interior que deben incorporar dinámicas de funcionamiento interno de los centros que garanticen los derechos reconocidos a la infancia en los tratados internacionales y la legislación nacional.

Todos estos procesos deben entenderse en un continuo cuya consecución pasa por distintos niveles y en cuyo desarrollo aparecen situaciones favorecedoras o que dificultan su implantación.

Revisaré a continuación cada uno de los puntos citados.

\section{3.a. Aplicación del criterio de normalización en los centros.}

El criterio de normalización debe entenderse en relación a:

- La utilización de la red ordinaria de servicios (Educación, Sanidad...) por los menores internos. Este aspecto podemos decir que está prácticamente cubierto, dado que es muy difícil encontrar centros que mantengan servicios internos para los menores atendidos. Sin embargo, habría que plantearse las condiciones en las que vienen siendo atendidos estos menores u otros que, sin estar en internados, requieren de una actuación compensatoria desde los recursos normalizados.

- Las condiciones estructurales que disponen los lugares de convivencia en los que se desarrolla el niño que permanece interno en 
una institución. Teóricamente, los equipamientos residenciales deberían ser lo más similares posibles a los que viven la mayoría de los niños, en cuanto a tamaño, emplazamiento, etc. Sin embargo, hemos visto como políticas de potenciación de uso de viviendas normalizadas como recursos de internamiento han fracasado fundamentalmente por criterios económicos.

- Las dinámicas de vida cotidiana deben asegurar un sistema de relaciones interpersonales lo más parecidas posibles a las del resto de los niños. Igualmente, nos encontramos aquí ante organizaciones institucionales especialmente rígidas que impiden aprendizajes que desarrollen niveles de autonomía personal suficiente.

\section{3.b. Mejora de la infraestructura de los centros.}

La infraestructura de los centros transferidos obedecía a unas finalidades que son totalmente diferentes a las que actualmente se pretenden. Para resolver esta cuestión ha sido necesario acometer remodelaciones internas que aseguraran la posibilidad de desarrollar programas educativos ajustados a las necesidades de los menores en ellas atendidas. Por ejemplo, reducir la capacidad de los dormitorios promoviendo capacidad individual o doble, adecuar el edificio a las necesidades de menores con dificultades de movilidad, etc.

\section{3.c. El establecimiento de condiciones mínimas para autorizar el funcionamiento de los centros.}

La necesidad de ordenar y diversificar los equipamientos residenciales dedicados a la protección de menores ha requerido que, en un primer momento, se establecieran la tipología de equipamientos institucionales, definir su funcionalidad, determinar sus prestaciones y establecer las dotaciones de personal (en número y cualificación) necesaria para un correcto funcionamiento. Estas serían las condiciones necesarias para que la Entidad Pública autorizara el funcionamiento de un centro de protección a la infancia.

\section{3.d. Diversificación de las tipologías de centros.}

Los centros de la Comunidad Valenciana han sido clasificados en función de la tarea a desarrollar y la edad de los usuarios en Centros de Recepción y Acogida, Residencias Comarcales, Residencias Infantiles, Residencias Juveniles y Centros de Reeducación.

Esta tipología de recursos deberá ser revisada, dado que no parece adecuado seguir manteniendo criterios como mantener en un mismo 
tipo de centros a menores de edades entre 6-18 años o resolver el debate de si pueden ser atendidos en los mismos centros menores de protección y reforma.

\section{3.e. La profesionalización de los centros vs. el voluntarismo}

Uno de los criterios en los que se ha basado la reorganización de los centros de infancia ha sido el mejorar la profesionalización de las prestaciones. En este proceso de profesionalización ha sido fundamental la situación de falta de reconocimiento y formación específica de la figura profesional del educador, que ha mantenido en una permanente e innecesaria situación de conflictividad a los centros de infancia. Este hecho, junto con la falta de definición del papel del voluntariado en los programas de atención a la infancia, provoca, en ocasiones, debates que no esconden, en mi opinión, sino intereses de tipo económico.

En este momento, la entrada en vigor de la Ley del Voluntariado y el reconocimiento como titulación universitaria de Grado Medio de los estudios de educador social crean las condiciones idóneas para acometer una ordenación eficaz que regule la participación del voluntariado de los centros de infancia sin necesidad de confrontación con la siempre deseable mejora de la profesionalización de los mismos.

Igualmente necesario, en mi opinión, es la incorporación de equipos técnicos a las residencias de menores, dado que el aumento de la complejidad de los casos allí atendidos así lo requiere.

Por último, hacer referencia a la necesidad de mantener sistemas de formación continua que posibiliten una adecuación de la práctica cotidiana a las necesidades de la población atendida.

\section{3.f. Incorporar en los centros dinámicas internas que aseguren el} ejercicio de los derechos reconocidos a la infancia.

El desarrollo de la normativa reguladora del funcionamiento de los centros, a través del Estatuto Básico de los Centros de Infancia y Juventud, la obligación de que los equipos profesionales definan objetivos generales de la institución, a través de los Proyectos Educativos, o que se establezcan las estrategias organizativas y de funcionamiento interno de los centros, a través de los Reglamentos de Régimen Interior, son los mecanismos utilizados para asegurar un sistema de garantías en el ejercicio de los derechos de los menores internos.

No obstante estos esfuerzos, es necesario seguir profundizando en la concreción de desarrollos normativos que aseguren sistemas de fun- 
cionamiento interno participativos, orientados a potenciar la autonomía personal, que tomen como objeto de intervención no sólo al niño sino, y fundamentalmente, a la familia, y, en definitiva, que aseguren de forma inequívoca el ejercicio de los derechos reconocidos a la infancia.

6.3.g.

Por último, me gustaría destacar la necesidad de encuadrar los equipamientos residenciales en la red de protección infantil, evitando su consideración como recurso aislado. Por el contrario, es necesario potenciar la creación de redes que permitan el desarrollo de procesos coherentes y globales de evaluación-atención y retorno a domicilio o a otra medida de protección de carácter permanente integrando recursos previstos en los niveles de atención primaria y especializada.

\subsection{Desarrollo de programas de acogimiento familiar.}

La medida de acogimiento familiar, entendida como el confiamiento de un menor a una familia distinta a la suya, para que le cuide y eduque, ha sido una de las formas de autoauyuda más antiguas como forma de atender a una familia que atraviesa dificultades.

Se reconoce como una medida de apoyo entre familias que disponen de una red social suficientemente amplia y dispuesta a prestar apoyo en momentos de crisis.

Sin embargo, hay familias que no cuentan con otros adultos en los que puedan confiar suficientemente cuando se encuentran en grave dificultad, por tanto, cuando se encuentran en situación de crisis, requieren de ser apoyados de forma temporal o permanente por otras familias ajenas a su ámbito.

En la evolución que ha seguido el acogimiento familiar, en lo relativo al procedimiento de formalización, se ha pasado de una forma de resolución, a partir del acuerdo mutuo entre partes, a ser necesaria la participación de la Entidad Pública, tanto en procesos de mutuo acuerdo cuando, sobre todo, no existe acuerdo.

La regulación normativa sobre el acogimiento familiar, así como su promoción, han ido unidos a la evaluación que, respecto de las consecuencias negativas de los internamientos de menores, se realizan a partir de la Segunda Guerra Mundial.

Así, en nuestro ordenamiento jurídico, podemos observar:

1. La escasa utilización, y relacionada en la mayoría de los casos con situaciones que no quedan abiertas a la adopción, cuando se apli- 
caba la normativa contenida en el texto refundido de la Ley de Tribunales Tutelares de Menores (1948). Nos referimos a la llamada colocación en familia.

2. El aumento del número de casos atendidos en acogimiento familiar temporal (con previsión de retorno) se produce a partir de 1980 con la implicación de las Entidades Públicas Municipales y de Comunidades Autónomas. En esta ocasión, se utiliza como recurso social dispuesto para la cobertura de un tipo de necesidades para las que no existía un dispositivo adecuado, dado que éstos se reducían a internamientos, adopciones o mantenimiento en domicilio. Las Comunidades Autónomas pioneras en este tipo de programas de Acogimiento Familiar Temporal fueron Cataluña (1983) y Valencia con el programa de Familias Educadoras (1986).

3 . Con la entrada en vigor de la Ley $21 / 87$, se introduce la figura del acogimiento familiar, siendo posible su formalización a partir de un procedimiento administrativo o judicial. Esto posibilita la aparición de distintas modalidades de acogimiento familiar conforme los desarrollos reglamentarios que aparecen en cada una de las Comunidades Autónomas.

Así, en la Comunidad Autónoma Valenciana se regulan las siguientes modalidades (Decreto 23/88) de acogimiento familiar:

a. El Acogimiento por una Familia Educadora: cuando se trate de desarrollar un proyecto educativo global y se pretenda el retorno del menor a su domicilio. La intervención de la Familia Educadora no se limita al acogimiento del menor sino que se compromete a apoyar procesos de recuperación de la familia biológica.

b. El acogimiento en los casos de suspensión de guarda y educación abiertos a la reinserción familiar.

c. Los acogimientos previos a la formalización de la adopción.

4. Tras la aplicación de estas modalidades de acogimiento familiar, se producen modificaciones importantes tanto a nivel de Comunidad Autónoma Valenciana (Ley 7/94 de la Infancia, Generalitat Valenciana) como muy recientemente las modificaciones incorporadas por la Ley Orgánica 1/96 de Protección Jurídica del Menor. Analizando ambos marcos legales, es posible desarrollar un cuadro comparativo a partir de los siguientes criterios:

- Instancia administrativa a quien compete su implantación.

- Objetivo y finalidad del acogimiento.

- Tipo de acogedor/es.

- Características del menor acogido y su familia.

- Organismo responsable de la Formalización y resolución.

- Tiempo previsto de duración. 
Teniendo en cuenta cada uno de ellos, paso a sintetizar, en los cuadros siguientes, las distintas modalidades de acogimiento actualmente vigentes, no sin antes dejar claro que podrían ser otras muchas variables a partir de las que es posible establecer una clasificación de los acogimientos familiares (naturaleza de la intervención, modalidad de formalización, compensación-retribuido o no retribuido, nivel de participación de la familia origen, etc.).

\subsection{Ampliación de programas de nivel especializado.}

La red de protección infantil necesita de un desarrollo de servicios especializados capaces de diversificar las prestaciones existentes y posibilitar una atención más individualizada a las necesidades infantiles dado que éstas se encuentran en permanente cambio y una estructura bipolarizada de servicios residenciales y de acogimiento familiar aparece como claramente insuficiente.

Esta ampliación de programas de nivel especializado no debe atender exclusivamente a la modalidad de intervención asistencial o rehabilitadora prevista para la atención a la infancia, sino que es indispensable su crecimiento en la modalidad de intervención de carácter preventivo.

En mi opinión, la idea que fundamenta la necesidad de crecimiento de este tipo de programas sería: Toda la legislación vigente en materia de protección a la infancia pone en juego dos principios que deben orientar la actuación de las Entidades Públicas. Estos principios, como ya hemos referido, son "el respeto al supremo interés del niño" y "el mantenimiento del menor en su medio familiar de origen salvo que no sea conveniente para su interés".

En la práctica, lograr que no entren en colisión ambos principios resulta, en ocasiones, muy dificil. Pensemos que limitar las posibilidades de actuación ante situaciones de desprotección infantil a la utilización de programas de atención primaria dispuestos para situaciones no especialmente complejas o a acogimientos familiares o residenciales supone la renuncia a poder atender conflictos que se producen en sistemas familiares multiproblemáticos, atribuyendo el calificativo de no recuperables a familias con las que no hemos sido capaces de desarrollar programas realmente especializados y adecuados a las necesidades planteadas que, asegurando la necesaria protección al niño/a permitan a su vez desarrollar un trabajo de intervención familiar que posibilite iniciar procesos de recuperabilidad de la familia, capacitándola para poder atender a su hijo, haciendo compatible los dos principios básicos de la protección infantil. 
Pero, ¿de qué tipo de programas estaríamos hablando?. Como he indicado anteriormente, sería necesario el desarrollo de estos programas desde las distintas modalidades de intervención, es decir, desde la prevención, la asistencia o la rehabilitación. Citaré algunos ejemplos que, en mi opinión, serían significativos.

DESDE LA PREVENCIÓN, sería deseable la incorporación y desarrollo de programas de mediación familiar. Pensemos que un gran número de los niños/as que se encuentran en situación de riesgo pertenecen a familias que se encuentran, o han pasado, por conflictos derivados de procesos de separación familiar o divorcio. Por este motivo, implantar de forma generalizada programas de mediación familiar, cuya finalidad primordial es el "evitar enfrentamientos inútiles entre la pareja y su incidencia negativa entre los menores, así como fomentar la coparentalidad", parece puede ampliar la red de programas eficaces para la prevención de procesos negativos que afecten al bienestar infantil. Esta modalidad de programas vienen siendo desarrollados en la Comunidad Autónoma de Madrid y, al parecer, las evaluaciones realizadas al respecto desprenden resultados satisfactorios.

DESDE LA ASISTENCIA. Nos enfrentamos, en esta segunda modalidad de intervención, ante situaciones que ya están produciendo un daño en el niño/a, provocando una situación de riesgo o desamparo. Es por ello necesario adoptar medidas que, asegurando la protección del niño/a, permitan desarrollar programas de recuperación familiar. En este sentido, parece muy interesantes experiencias que se han desarrollado tanto en nuestro país como en el extranjero. Me refiero a programas que combinan, simultáneamente, la protección del niño/a, asegurando dispositivos de separación provisional del núcleo familiar biológico (bien en acogimiento residencial o familiar) y programas de intervención (o terapia) con la familia biológica. Cito ejemplos como el Centro para el niño maltratado y el tratamiento de la familia en crisis de Milán o los programas de acogimiento familiar especializado con finalidad de reintegración familiar.

DESDE LA REHABILITACIÓN. Trataríamos, por último, de atender situaciones que, habiendo pasado por procesos intensos de crisis, requieren de apoyos para poder retomar procesos normalizadores de vida cotidiana. Dentro de este tipo de programas, merecería especial importancia los de terapia familiar, llevados a cabo de forma simultánea con programas de incorporación al mundo laboral u otros programas destinados a atender problemáticas específicas de alguno/s miem$\mathrm{bro} / \mathrm{s}$ de la unidad de convivencia (tratamientos de salud, drogodependencias, etc.). 


\section{VII.LA ACTUACIÓN DE LA ENTIDAD PÚBLICA EN MATERIA DE EJECUCIÓN DE MEDIDAS DICTADAS POR LOS JUZGA- DOS DE MENORES.}

Con la entrada en vigor de la Ley Orgánica 4/92 de 5 de junio, sobre reforma de la Ley Reguladora de la competencia y el procedimiento de los Juzgados de Menores, surgida como consecuencia de la Sentencia 36/1991 del Tribunal Constitucional, por la que se declaraba inconstitucional el art. 15 de la Ley de Tribunales Tutelares de Menores de 1948, se viene a cubrir la falta de un procedimiento específico adaptado a la situación del menor, pero en absoluto supone una reforma en profundidad del sistema de Justicia Juvenil existente en nuestro país.

No obstante, la Ley Orgánica 4/92 introduce importantes modificaciones, como, por ejemplo, las siguientes:

- Atribución al Ministerio Fiscal de la defensa de los derechos, la observancia de las garantías y el cuidado de la integridad física y moral del menor.

- Atribución al Ministerio Fiscal la instrucción de los expedientes de los menores a los que se le impute la comisión de un delito o falta.

- Reconocimiento a los menores de los derechos que se establecen en la Ley de Enjuiciamiento Criminal para los adultos.

- Obligatoriedad de requerimiento de un informe sobre situación psicológica, educativa, familiar y social del menor, en el momento resulte imputado.

En relación al papel de la Entidad Pública en la jurisdicción de menores, cabe decir que la Disposición Adicional Tercera de la Ley Orgánica 4/92 dice textualmente: «La ejecución de las medidas adoptadas por los Juzgados de Menores corresponde a las Entidades Públicas competentes en la materia».

Igualmente, se atribuye competencia a las Entidades Públicas en cuanto a la adopción de medidas educativas y formativas respecto de: 1. Menores de 12 años que hubieran cometido delitos o faltas según las leyes penales.

2. Mayores de 12 años y menores de 16 que, habiendo cometido un delito o falta, el Juez de Menores determine su remisión a las Instituciones administrativas, siempre y cuando en la comisión de los hechos no se hubiera utilizado grave violencia o intimidación. 


\section{VIII.PROGRAMAS DE REINSERCIÓN Y EJECUCIÓN DE MEDI- DAS DICTADAS POR LOS JUZGADOS DE MENORES.}

Para la ejecución de las medidas que determina el Juez de Menores, conforme a la Ley Orgánica 4/92, la Generalitat Valenciana ha diseñado los Programas de Reinserción previstos en Ley 7/94 de la Infancia.

Los medidas previstas dentro de los Programas de Reinserción son las siguientes:

A. De Atención Primaria (Competencia municipal).

1. La amonestación.

2. La libertad vigilada.

3. El asesoramiento educativo, terapeútico y ocupacional.

4. La prestación de servicios en beneficio de la comunidad.

5. El internamiento de uno a tres fines de semana.

6. La reparación extrajudicial.

B. Alternativas a la ejecución del nivel primario (competencia municipal).

1. La instrucción de apoyo.

2. La instrucción de tratamiento terapéutico.

3. El asesoramiento educativo.

C. De atención Especializada (Competencia de la Generalitat).

1. El acogimiento por otra persona o núcleo familiar.

2. El tratamiento ambulatorio.

3. El ingreso en un centro de carácter terapéutico.

4. El ingreso en un centro en régimen abierto, semiabierto o cerrado.

La no definición de las medidas en la Ley 4/92 provoca que se definan en la Ley 7/94 de la Infancia, pero esta definición es, también parcial, lo que podría suponer inseguridad jurídica para los menores.

A lo largo de estos cinco años de aplicación de la L.O. 4/92, la imposición de las medidas impuestas por los Juzgados de Menores ha estado fuertemente bipolarizada, utilizándose, fundamentalmente, las de libertad vigilada y la de internamiento en sus distintos regímenes, siendo el tratamiento ambulatorio una medida que se adopta con frecuencia, acompañando a la medida de internamiento. No obstante, es de destacar, en el último año, el aumento de la imposición de medidas de prestación de servicios en beneficio de la comunidad.

Del resto de las medidas, cabría indicar que algunas se han utilizado en muy pocas ocasiones, como, por ejemplo, la reparación extrajudicial, las de acogimiento familiar. 
Algunas de estas medidas, no desarrolladas suficientemente, sí lo han sido en otras Comunidades Autónomas, siendo evaluadas como altamente eficaces. La más destacable, sin duda, son los resultados que están siendo obtenidos con la implantación de servicios de mediación extrajudicial, cuyo objetivo es lograr un tratamiento del menor infractor por fuera de lo que supone la judicialización del expediente.

\section{IX.ORGANIZACIÓN DE RECURSOS PARA LA EJECUCIÓN DE MEDIDAS JUDICIALES}

La Generalitat Valenciana, desde el momento que asume las competencias en esta materia, ha ido promoviendo la organización de recursos que garantizaran el cumplimiento de su responsabilidad.

Así, de forma progresiva, se han ido incorporando profesionales a los Equipos Sociales de Base Municipales que se encargan directamente de ejecutar los programas educativos que dan contenido a las medidas judiciales de orden abierto (libertad vigilada, prestación de servicios en beneficio de la Comunidad, etc.).

El número de profesionales que actualmente están asignados a Equipos Base Municipales de la Comunidad Valenciana lo han hecho en dos modalidades: la primera, ampliándose los equipos de atención primaria, la segunda, creando o concertando con Entidades sin ánimo de lucro servicios que específicamente se dedicaran a la ejecución de estas medidas.

Los recursos utilizados para la ejecución de medidas de orden abierto son, en la mayoría de las ocasiones, los ya existentes en las localidades de residencia de los menores, promoviendo un criterio de normalización e intentando, a su vez, incorporar las adaptaciones individuales que permitan adecuar los programas educativos a las necesidades de los menores.

En relación al cumplimiento de las medidas de prestación de servicios en beneficio de la comunidad, se utilizan, como regla general, la incorporación de los jóvenes a actividades que tengan un beneficio de interés general y social, promoviendo la toma de conciencia del joven sobre las necesidades de otras personas.

La medida de tratamiento ambulatorio, en la mayoría de las ocasiones, ha sido utilizada para la atención a jóvenes con problemas de drogadicción, y unidas a otra medida de internamiento en sus distintas modalidades. 
Esto ha supuesto, por una parte, la apertura de los Centros de Atención a Drogodependientes a casos de menores de 16 años (población que quedaba fuera de los tratamientos ofertados por estos centros) y, por otro lado, que el apoyo educativo en el proceso de tratamiento de la drogadicción deba ser prestado desde los centros de internamiento.

Las medidas de internamiento han sido llevadas a cabo en Residencias Comarcales o en Centros de Reeducación. En los primeros, se ejecutan básicamente las medidas de internamiento en régimen abierto y semiabierto, en los segundos, las medidas de internamiento en régimen semiabierto (reincidentes) y cerrado.

Cuando es necesario la elaboración de informes de diagnóstico en régimen de internamiento abierto o semiabierto, se utiliza el Centro de Recepción.

Tanto el Centro de Recepción como las Residencias Comarcales son establecimientos desde donde se atienden a menores que se encuentran bajo protección de la Administración o cumpliendo una medida impuesta por el Juzgado de Menores.

En los Centros de Reeducación, se atienden menores que se encuentran cumpliendo medida impuesta por los Juzgados de Menores.

La medida de ingreso en un centro terapéutico supone una dificultad importante para su ejecución, dado que, tanto para el tratamiento de problemas de drogadicción como problemas de salud mental, no existen recursos específicos para la atención a jóvenes de edades inferiores a 16 años.

La carencia de centros específicos conlleva, en ocasiones, la necesidad de llevar a cabo la medida en Residencias Comarcales o Centros de Reeducación, buscando apoyos ambulatorios que garanticen un tratamiento lo más adecuado posible.

Me gustaría destacar aquí la importancia de establecer equipos de asesoramientos a la Fiscalía y Juzgado de Menores, suficientes en cuanto a su número y capaces de superar la línea del puro informe de carácter pericial, llegando a configurar modelos de diagnóstico a partir de los cuales puedan desarrollarse programas educativos individuales que den sentido a la medida judicial impuesta. En este sentido, parece necesario potenciar procedimientos que puedan acercar evaluación y atención como partes de un mismo proceso.

Por último, reseñar la importancia que, para el cumplimiento de las medidas impuestas por Juzgados de Menores, va a tener la elevación de la mayoría de edad a los 18 años, ordenada en nuestro nuevo Código Penal, dado que, para la correcta atención a estos jóvenes, se requerirá no sólo de un aumento de los recursos y prestaciones dispo- 
nibles en la actualidad sino una mejora sustancial en la preparación de los profesionales a los que corresponda su atención directa.

Como conclusión de todo lo dicho, quisiera destacar lo que, en mi opinión, es la clave para asumir el reto de ir adecuando la estructura de prestaciones y servicios dispuestos para la protección a la infancia y juventud en nuestra Comunidad Autónoma. Parece imprescindible evaluar los cambios que respecto de las necesidades de la infancia y juventud se han producido en los últimos años y relacionarlos con el sistema legal actualmente vigente, a fin de promover cambios en la estructura de servicios y formación de personal que posibilite un ajuste entre prestaciones y necesidades a atender.

Esta revisión debe ser incorporada al método de trabajo, dado que, de no hacerlo, podría volver a producirse una situación como la actual en la que no se analiza en profundidad el funcionamiento de los recursos, produciéndose un desajuste que, por una parte, genera insatisfacción en los profesionales y, por otra, y lo que es más importante, genera una inadecuada respuesta ante las problemáticas de la infancia y la juventud.

Un excelente momento para adoptar esta medida sería el desarroIlo del Plan Integral de Atención a la Infancia previsto en la ley de la Infancia de la Comunidad Valenciana del año 1994 que, en estas fechas, sigue sin ser desarrollado.

\section{BIBLIOGRAFÍA}

ACTAS DEL IV CONGRESO DE INFANCIA MALTRATADA: Calidad y Eficacia como metas. Sevilla, del 8 al 11 de noviembre de 1995.

BARJAU CAPDEVILA, C.: El acogimiento Familiar, un medio de protección infantil. En Manual de Protección Infantil. Coordina Joaquín de Paul Ochotorena. Edit. Masson, 1995.

CASAS AZNAR, F.: Instituciones Residenciales ¿Hacia dónde?. III Congreso Estatal sobre Infancia Maltratada, 1993.

COLTON, M.J. y HELLINCKX, W.: La Atención a la Infancia en la Unión Europea. Ministerio de Asuntos Sociales, 1993.

ECHEVESTE ALBAITERO, J.A.: Reseña normativa en materia de protección de menores. En Intervención Social con Menores. Coordinado por Agustín Bueno. Edita Universidad de Alicante y Fundación Cultural CAM, 1996.

Estatuto Básico de Centros de Atención a la Infancia.

FUNES Y ARTIAGA, J.: Mediación y Justicia Juvenil. Generalitat de Catalunya. Colección Justicia y Sociedad,1994. 
Ley 5/97 de Servicios Sociales de la Generalitat Valenciana.

Ley 7/94 de la Infancia de la Generalitat Valenciana.

Ley Orgánica 1/96 de Protección Jurídica del Menor.

Ley Orgánica 4/92 sobre reforma de la Ley reguladora de la competencia y procedimientos de los Juzgados de Menores. 